\title{
Rendezvous de espaçonaves em órbitas próximas à Terra
}

\author{
Rendezvous of near earth orbiting spacecraft
}

\author{
Iram Gleria*
}

Instituto de Física, Universidade Federal de Alagoas, Maceió, AL, Brasil

Recebido em 14 de Outubro, 2016. Revisado em 27 de Novembro, 2016. Aceito em 27 de Novembro, 2016.

No início dos anos sessenta, a corrida espacial entre a agência espacial americana NASA e o correspondente programa soviético envolveu a solução de diversos problemas desafiadores. Um deles era a habilidade de rendezvous (acoplamento) entre duas espaçonaves em órbita. O primeiro rendezvous foi efetuado pelos americanos na missão Gemini 8, comandada por Neil Armstrong, que posteriormente viria a ser o primeiro homem a pisar na Lua. Dentre os vários aspectos interessantes do problema do acoplamento em órbita, citamos o seguinte resultado paradoxal: considerando duas espaçonaves em uma mesma órbita próxima à Terra, se uma delas acionar seus foguetes com o intuito de aumentar a velocidade para alcançar a segunda espaçonave, acabará por ficar mais lenta. O objetivo deste artigo é analisar tais resultados, que resultam do fato de que altitude e velocidade são correlacionadas quando consideramos as equações de corpos em queda livre de acordo com as leis da gravitação.

Palavras-chave: gravitação universal, corrida espacial, manobra Hohmann.

In the early sixties, the space race between the American agency NASA and its Soviet counterpart involved the solution of several challenges. One of them was the ability of rendezvous between two orbiting spacecrafts. The Americans did the first successful rendezvous in the Gemini 8 mission commanded by Neil Armstrong, who years later would become the first man to set foot on the moon. Among the several interesting aspects of manned orbital rendezvous we cite the following paradox: considering two spacecrafts in the same near earth orbit, if one of them accelerates in order to catch up with the other, it will ultimately left behind. In this paper we analyze such counter intuitive results, which follows from the fact that altitude and velocity are correlated when we consider the equations governing free falling bodies, according Newton's Universal Gravitational Law.

Keywords: law of universal gravitation, space race, Hohmann transfer.

\section{Introdução}

A guerra fria foi um dos acontecimentos marcantes da segunda metade do século XX. As duas superpotências da época, EUA e URSS, disputavam entre si a hegemonia do mundo ocidental, contrapondo suas ideologias. Um palco onde a guerra fria se tornou especialmente interessante foi a corrida espacial. Uma extensão óbvia da corrida pela supremacia aérea, iniciada logo após o fim da segunda grande guerra, a corrida espacial possui como marco inicial o lançamento, em 04 de Outubro de 1957, do primeiro satélite artificial soviético Sputnik. Para os russos, o feito demonstrava o avançado nível da tecnologia

*Endereço de correspondência: iram@fis.ufal.br. soviética [1]. Os americanos tentaram uma resposta imediata, e em Dezembro do mesmo ano fizeram tentativas frustradas de colocar seu próprio satélite em órbita, que explodiu poucos segundos após o lançamento. Um fiasco que rendeu bons momentos à mídia americana, que o reportou em matérias de primeira página onde o fracassado satélite recebeu nomes como Flopnik e Kaputnik [1].

Os soviéticos mantiveram a dianteira por algum tempo. Seu programa espacial, chefiado por Sergei Pavlovich Korolev (cuja identidade os americanos desconheciam) e situado em Zvyozdny Gorodok (hoje conhecida no mundo ocidental pelo nome de Star City) logo se destacou por outros feitos. Enviou o primeiro ser vivo ao espaço, a cadela Laika, a bordo 
do Sputnik 2. Após dois outros vôos tripulados por cães enviou, em 12 de Abril de 1961, o primeiro homem ao espaço: o cosmonauta Yuri Gagarin, a bordo do módulo Vostok 1. Gagarin completou uma órbita quase completa em volta da Terra, sobrevoando o Japão, o Oceano Pacífico, a América do Sul e o Atlântico. Acionou seus foguetes sobre a África e desceu sobrevoando a Turquia [2] O programa espacial americano tentava correr atrás do prejuízo. Em 1959 havia selecionado o grupo pioneiro de astronautas para as missões Mercury, através da recém criada agência NASA (National Aeronautics and Space Administration), que em 1958 substituiu a NACA (National Advisory Committee for Aeronautics), fundada em 1917 [2] Em 05 de Maio de 1961, pouco tempo após o vôo de Gagarin, os americanos colocaram Alan Sheppard no primeiro vôo espacial americano, um feito muito aquém do soviético, já que esse primeiro vôo Mercury foi apenas suborbital (um grande arco de parábola e não uma órbita elíptica) durando cerca de 15 minutos, o que não impediu Alan Sheppard de se tornar uma celebridade instantânea [3].

Um aspecto interessante das primeiras missões espaciais era o altíssimo grau de detalhes técnicos que deveriam ser observados. Considere por exemplo as primeiras tentativas de EVA, ou Extra Vehicular Activity, em que o astronauta deveria sair de seu veículo e realizar alguma atividade no vácuo do espaço. Naturalmente, todos que estavam envolvidos conheciam o princípio de conservação do momento linear e sabiam das dificuldades de estarem em um meio que não oferece nenhuma resistência, dificultando tarefas triviais como por exemplo interromper um movimento relativo de afastamento da nave ou realizar trabalhos mecânicos que envolviam movimentos de tração, rotação etc [3, 4]. Os russos foram pioneiros em realizar um EVA, com Alexei Leonov na missão Voskhod 2. A EVA foi feita com o cosmonauta ligado a nave por um umbilical, tanto para impedir o caminhante de se perder de maneira irreversível no espaço como para garantir suprimento elétrico etc. Para impedir eventuais embaraços do umbilical, a nave estava impedida de movimentos rotatórios, o que a submetia a extremos níveis de temperatura no lado exposto ao Sol. A EVA de Alexei Leonov durou pouco mais de 12 minutos e, na altitude em que se encontravam não havia (praticamente nenhuma) atmosfera para absorver parte da radiação solar, principal- mente raios UV, assim o lado da nave exposto ao Sol atingiu rapidamente temperaturas muito altas enquanto no lado da sombra havia temperaturas extremamente baixas. Segundo Leonov, isso pode ter causado uma deformação que levou a um vazamento dentro da espaçonave. Os sistemas automatizados da nave compensaram lançando uma quantidade excessiva de oxigênio, e houve o risco de explosão se uma fagulha ou faísca aparecesse nos circuitos, um tipo de acidente que acabou por realmente acontecer anos depois com a Apollo 1, que trabalhava em um ambiente contendo $100 \%$ de $O_{2}$, vitimando toda sua tripulação com a nave ainda em solo. Naturalmente, como o mesmo problema de extremos de temperatura poderia atingir o astronauta, o traje espacial continha múltiplas camadas de isolamento para impedí-lo de ser literalmente fritado ou congelado dependendo do lado que estava exposto ao Sol. $\mathrm{O}$ astronauta Eugene Cernan, o primeiro americano a realizar um EVA, a bordo da Gemini 9A, teve problemas com as várias camadas de isolamento térmico de seu traje espacial, que durante sua EVA se tornou tão rígido que dificultou os trabalhos previstos e quase resultou em uma fatalidade. Eugene Cernan viria posteriormente a ser o último homem a pisar em solo lunar, como comandante da Apollo 17, em 1972 [5,6].

O primeiro rendezvous ocorreu em Março de 1966, na missão Gemini 8, a sexta missão tripulada dentro do programa Gemini. Comandanda por Neil Armstrong, foi a décima segunda missão tripulada lançada pela NASA (seis missões no programa Mercury e outras seis no programa Gemini), e marca o ponto em que os americanos ultrapassaram os russos na corrida espacial, que foram pioneiros em colocar o primeiro satélite em órbita, depois um ser vivo (Laika) e finalmente um homem (Yuri Gagarin) e em efetuar um EVA com Alexei Leonov. Armstrong acoplou seu módulo de vôo ao foguete não-tripulado Agena lançado previamente, um feito que (novamente) quase acaba em tragédia. Após o acoplamento a cápsula e o foguete Agena começaram um perigoso movimento rotatório, o que forçou um desacoplamento prematuro. Entretanto, isso não foi suficiente para conter o giro, que posteriormente revelou-se ser devido a um propulsor defeituoso da Gemini. Para conter o giro, os astronautas foram obrigados a utilizar outros propulsores, destinados a estabilizar a reentrada na atmosfera, e a missão teve que ser encerrada dez horas após seu início [3]. 
O primeiro rendezvous soviético aconteceria apenas em Janeiro de 1969 com as naves Soyuz 4 e 5. Como as duas naves eram tripuladas, os soviéticos foram pioneiros no acoplamento de duas espaçonaves tripuladas. Vladimir Shatalov decolou na Soyuz 4, enquanto Yevgeny Khrunov, Aleksei Yeliseyev e Boris Volynov na Soyuz 5. Os americanos repetiriam o feito com a Apollo 9 em março do mesmo ano [2].

Neste artigo vamos analisar, com auxílio da lei da gravitação de Newton, alguns aspectos físicos envolvidos no acoplamento de espaçonaves em órbita, fixando nossos exemplos em duas manobras bem conhecidas: a manobra Hohmann e a transferência bielíptica. Antes de continuarmos, é bom lembrarmos que, tanto nos idos de 1970 quanto no atual estágio da tecnologia espacial, praticamente todo o combustível disponível se exaure nos estágios de lançamento, sendo essencialmente utilizado para a espaçonave entrar em órbita (ou, nas missões lunares, em uma manobra que os livrava da gravidade da Terra e os colocaria em rota para a Lua, denominada Trans Lunar Injection). O que resta pode ser utilizado em pequenas correções de órbitas, mudanças de atitude da nave e, principalmente, na reentrada da atmosfera. Assim sendo, os dois corpos que pretendem ser acoplados estão em queda livre, com uma fonte de energia que pode ser acionada apenas por poucos segundos, fazendo com que o corpo saia de uma órbita e entre em outra.

\section{Gravitação Universal}

A importância do problema de rendezvous para o programa espacial pode ser ilustrada com o recrutamento do astronauta E. E. Audrin para o programa Gemini da NASA. Também conhecido por Buzz Aldrin, era um piloto da força aérea que, em 1963, defendeu no MIT uma tese de doutorado intitulada Line of sight guidance techniques for manned orbital rendez-vous, que tratava do problema de acoplamento não coplanar (os objetos que desejamos acoplar possuem órbitas em planos distintos) [7]. A tese apresentava um método para se determinar as mudanças de velocidade requeridas para o acoplamento ocorrer em um determinado ponto, a partir de duas medidas feitas sobre o objeto alvo (o objeto que se deseja acoplar). Pouco tempo depois, foi selecionado pela NASA como astronauta, pesando em seu curriculum o trabalho sobre rendezvous. Em 1969 se tornou (razoavelmente) famoso por ter sido o segundo homem a pisar na Lua. Algumas de suas idéias sobre acoplamento foram efetivamente utilizadas nos programas Gemini e Apollo.

Vamos revisar os conceitos básicos envolvidos no problema do rendezvous, que está relacionado ao problema de dois corpos em uma força central [8]. Considere um referencial inercial, nos quais os corpos possuem coordenadas $\overrightarrow{r_{1}}$ e $\overrightarrow{r_{2}}$. Usualmente escolhemos tratar o problema a partir da posição do centro de massa do sistema $\vec{R}$ e da distância relativa $\overrightarrow{r_{1}}-\overrightarrow{r_{2}}$. No problema de força central em que não há atritos a energia potencial é função de $r=\left|\overrightarrow{r_{1}}-\overrightarrow{r_{2}}\right|$ com lagrangeana:

$$
L=\frac{1}{2} \mu\left(\dot{r}^{2}+r^{2} \dot{\theta}^{2}\right)-U(r)
$$

Onde escolhemos $\vec{R}=0, \mu=\frac{m_{1} m_{2}}{m_{1}+m_{2}}$ é a massa reduzida e usamos coordenadas polares. Como $\theta$ é coordenada cíclica, o momento conjugado (momento angular) se conserva: $p_{\theta}=\frac{\partial L}{\partial \dot{\theta}}=\mu r^{2} \dot{\theta}=$ cte. Que é uma forma de expressar a Segunda Lei de Kepler, também conhecida por lei das áreas. De fato, veja na Figura 1 que o vetor $\vec{r}$ percorre uma área $d A=\frac{r^{2} d \theta}{2}$ em um tempo $d t$, então: $\frac{d A}{d t}=\frac{r^{2} \dot{\theta}}{2}=\frac{p_{\theta}}{2 \mu}=c t e$

Além do momento angular, uma outra grandeza obviamente conservada é a energia:

$$
E=T+U=\frac{\mu \dot{r}^{2}}{2}+\frac{p_{\theta}^{2}}{2 \mu r^{2}}+U(r)
$$

A partir desta relação podemos expressar as órbitas em temos de $r$ e $\theta$. Com $d \theta=\frac{d \theta}{d t} \frac{d t}{d r} d r$ e usando $\dot{\theta}=p_{\theta} / \mu r^{2}$ e $\dot{r}$ obtido de 2 .

$$
\theta(r)=\int \frac{ \pm\left(p_{\theta} / r^{2}\right) d r}{\sqrt{2 \mu\left(E-U-p_{\theta}^{2} / 2 \mu r^{2}\right)}}
$$

Com $U=-G m_{1} m_{2} / r$ para corpos sob influência da força da gravidade, $G=6,67 \cdot 10^{-11} \mathrm{~m}^{3} /\left(\mathrm{Kg}_{\mathrm{g}} \mathrm{s}^{2}\right)$.

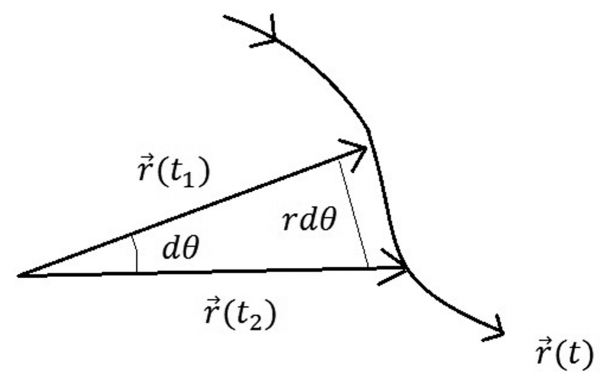

Figura 1: Segunda Lei de Kepler a partir da varredura do vetor $\vec{r}$ no tempo $d t$. 
Tabela 1: Órbitas no problema de dois corpos sob atração gravitacional.

\begin{tabular}{lcc}
\hline Excentricidade & Energia & Seção Cônica \\
\hline$\epsilon>1$ & $E>0$ & Hipérbola \\
$\epsilon=1$ & $E=0$ & Parábola \\
$0<\epsilon<1$ & $V_{\min }<E<0$ & Elipse \\
$\epsilon=0$ & $E=V_{\min }$ & Círculo \\
\hline
\end{tabular}

Nesse caso a integral (3) pode ser resolvida analiticamente e a solução será [8]:

$$
\frac{\alpha}{r}=1+\epsilon \cos \theta
$$

$$
\text { onde } \alpha=\frac{p_{\theta}^{2}}{\mu k} \text { e } \epsilon=\sqrt{1+\frac{2 E p_{\theta}^{2}}{\mu G m_{1} m_{2}}}
$$

A equação (4) é uma seção cônica com $\epsilon$ sendo a excentricidade da órbita e $2 \alpha$ denominada latus rectum. Uma seção cônica é uma curva obtida a partir da intersecção de um plano e um cone de base circular. Dependendo da orientação entre o plano e o cone podemos obter uma curva fechada ou aberta, sendo as curvas fechadas elipses e círculos e as curvas abertas hipérboles e parábolas.

O menor valor de $r$ ocorre em $\dot{\theta}=0$ denominado pericentro (perigeu para corpos orbitando a Terra e periélio para corpos orbitando o Sol). Sendo o máximo valor de $r$ denominado apocentro (correspondentemente apogeu e afélio). Esses dois pontos são chamados genericamente de apsides das órbitas. De acordo com a excentricidade temos quatro casos possíveis, dados na Tabela 1 abaixo.

$\mathrm{Na}$ Tabela $1 V_{\min }=-\left(\frac{\mu G^{2} m_{1}^{2} m_{2}^{2}}{2 p_{\theta}^{2}}\right)$ é o menor valor possível da energia potencial que garante $\dot{r}^{2}>$ 0 . Note que corpos em órbitas fechadas (elipses e círculos) possuem energia total negativa. Os semieixos maior e menor de uma órbita elíptica são dados por, respectivamente [8]:

$$
\begin{aligned}
& a=\frac{2 \alpha}{1-\epsilon^{2}}=\frac{G m_{1} m_{2}}{2|E|} \\
& b=\frac{2 \alpha}{\sqrt{1-\epsilon^{2}}}=\frac{p_{\theta}}{2 \mu|E|}
\end{aligned}
$$

$\mathrm{O}$ pericentro da órbita ocorre em $r_{\min }=a(1-\epsilon)$ e o apocentro em $r_{\max }=a(1+\epsilon)$. O tempo que o corpo leva para descrever uma área completa da elipse é, naturalmente, o período $\tau$ do movimento, que podemos mostrar ser igual a 8$]$ :

$$
\tau^{2}=\frac{4 \pi^{2} a^{3}}{G\left(m_{1}+m_{2}\right)}
$$

que é a expressão da Terceira Lei de Kepler (apesar de Kepler ter anunciado a lei com a aproximação $m_{1}+m_{2} \approx m_{2}$, o que pode ser considerado se um dos corpos for o Sol, muito mais massivo que os planetas de nosso sistema solar, exceto, talvez, Júpiter).

Na superfície da Terra, o rendezvous entre dois objetos (dois automóveis ou dois navios por exemplo) envolve apenas especificar um local, a partir da Latitude e Longitude, e instruir os móveis a se deslocarem para lá, com velocidades específicas para atingirem o ponto de encontro no mesmo instante $t$ [9]. Para corpos em órbita, o local de encontro não é apenas um determindado ponto na superfície da Terra, mas também um ponto em uma altitude pré determinada. Além do mais, como dissemos acima, os corpos estão em queda livre e continuarão assim após o rendezvous, não podendo simplesmente $p a$ rarem após o encontro. Um corpo de massa $m$ em órbita circular a uma altitude de $400 \mathrm{Km}$ acima da Terra (aproximadamente a órbita da estação espacial ISS) possue uma velocidade de:

$$
\frac{G M_{T} m}{r^{2}}=\frac{m v^{2}}{r} \Rightarrow v=\sqrt{\frac{G M_{T}}{R}} \approx 7675 \mathrm{~m} / \mathrm{s}
$$

onde $M_{T} \approx 5,99.10^{24} \mathrm{Kg}$ é a massa da terra e $R=R_{T}+h, R_{T} \approx 6.371 \mathrm{Km}$ é o raio da Terra e $h=400 \mathrm{Km}$ nesse exemplo. Um erro de um segundo faz com que as espaçonaves "errem" o rendezvous por mais de $7 \mathrm{Km}$. A expressão (6) nos mostra a origem do paradoxo mencionado no abstract do artigo, pois, de acordo com ela, devemos viajar mais rápido numa órbita mais baixa. Para irmos para uma órbita mais alta, usamos nossos foguetes para acelerar, ganhar energia cinética e, consequentemente, irmos para uma órbita mais alta. De fato, para uma órbita circular em torno da Terra teremos $E=m v^{2} / 2-G m M_{T} / r$ e um acréscimo na energia cinética corresponde a um acréscimo na energia total. Como $E=-G m M_{T} / 2 r$ para órbitas circulares o novo equilíbrio será atingido em uma órbita mais alta ( $E$ maior mas negativo implica $r$ maior). Mas de acordo com (6) na órbita mais alta teremos uma velocidade menor que a que tínhamos antes de acionarmos os foguetes. Dessa forma ao acelerarmos a nave ela acaba por ficar, de certa forma, mais lenta. Entretanto, tais manobras são exatamente as que devem ser executadas para se conseguir o rendezvous. Sejam duas espaçonave na mesma órbita circular. A que está "atrás" pode 
"frear" e ir assim para uma órbita mais baixa. Nessa órbita ela viajará com maior velocidade e acabará por alcançar a outra. Após isso ela pode realizar manobras para ajustar a altitude correta para o rendezvous.

Seja o ângulo entre a velocidade tangencial da espaçonave $\vec{v}$ e a vertical local $\vec{r}$, onde $r=R_{t}+h$, dado por $\gamma$ (Figura 2). Da segunda lei de Kepler, como $\dot{\theta} r^{2}=$ cte e $v \sin \gamma=\dot{\theta} r$ teremos $v r \sin \gamma=$ cte. Considere a posição inicial $\gamma_{0}, v_{0}, r_{0}$ dados no instante em que os foguetes exaurem o combustível e a nave entra em queda livre. No apogeu e no perigeu $\gamma=90^{\circ}$ então $r_{p} v_{p}=r_{a} v_{a}=r_{0} v_{0} \sin \gamma_{0}$ onde $r_{p}$ designa o raio do perigeu (semi eixo menor), $v_{p}$ sua velocidade e $r_{a}, v_{a}$ o equivalente para o apogeu. Note que essa relação, junto com a conservação da energia mecânica permite-nos determinar $r_{p}$ e $r_{a}$ em termos de $\gamma_{0}, v_{0}, r_{0}$. Por sua vez, essas relações e mais (5) permite-nos escrever a excentricidade da órbita em termos de $\gamma_{0}, v_{0}, r_{0}$ :

$$
e=\sqrt{\left(\frac{r_{0} v_{0}}{G M_{T}}-1\right)^{2} \sin ^{2} \gamma_{0}+\cos ^{2} \gamma_{0}}
$$

O mesmo podendo ser feito para o latus rectum.

Conhecidos os parâmetros da órbita em termos das condições iniciais podemos partir para as manobras a serem realizadas durante um rendezvous 10 . O caso mais simples ocorre quando o segundo objeto não realiza nenhuma manobra, apenas o primeiro altera sua trajetória, que pode ser uma mudança na altitude ou plano orbital (a inclinação I da órbita é a distância angular do plano orbital e o equador terrestre). Esse foi o tipo de acoplamento realizado pela Gemini 8 com o foguete Agena, em 1966. Consideramos que as manobras de mudança de órbita são impulsivas, no sentido que um breve impulso é dado na velocidade da nave, durante o qual ela pode ser considerada em repouso $(\Delta t \rightarrow 0)$. Assim, uma

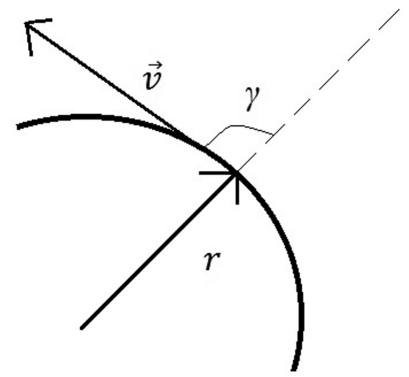

Figura 2: Relação entre a velocidade tangencial e a vertical local. manobra de passagem de uma órbita para outra que visa interceptar o segundo objeto, deve ser feita no ponto de intersecção das duas. Tais manobras envolvem mudanças de altitude ou inclinação. A maneira mais energeticamente eficiente de mudar a altitude de uma órbita é através de uma manobra Hohmann [8. A manobra Hohmann realiza dois impulsos, conforme visto na figura 3. Considere a primeira nave em uma órbita circular de altitude $r_{1 i}=R_{t}+h_{i}$ sendo que o objeto alvo que desejamos acoplar se encontra em uma órbita circular de raio $r_{1 f}$. Em um determinado ponto damos um impulso $\Delta v$ na mesma direção da velocidade orbital que leva a nave à uma órbita elíptica cujo apogeu ocorre em $r_{1 f}$. O semi eixo maior dessa elipse é $a=\left(r_{1 i}+r_{1 f}\right) / 2$ (vide Figura 3 ). Na órbita circular $r_{1 i}$ a energia será $E=-k / 2 r_{1 i}=m v_{1 i}^{2} / 2-k / r_{1 i}$ que nos leva a $v_{1 i}=\sqrt{k / m r_{1 i}}$, onde $k=G M_{T}$. O impulso $\Delta v$ deve levar a nave à órbita elíptica, onde

$$
E=-\frac{k}{r_{1 i}+r_{1 f}}=m v_{e}^{2} / 2-\frac{k}{r_{1 i}}
$$

que nos leva a

$$
v_{e}=\sqrt{\frac{2 k}{m} \frac{r_{1 f}}{r_{1 i}\left(r_{1 i}+r_{1 f}\right)}}
$$

de modo que o impulso $\Delta v=v_{e}-v_{1 i}$ pode ser calculado em termos dos parâmetros da órbita. A nave continua nessa órbita, chamada phasing orbit e que se dá em um elipse de transferência até interceptar a órbita $r_{1 f}$, onde devemos realizar outro impulso para sairmos da órbita elíptica e entrarmos na órbita

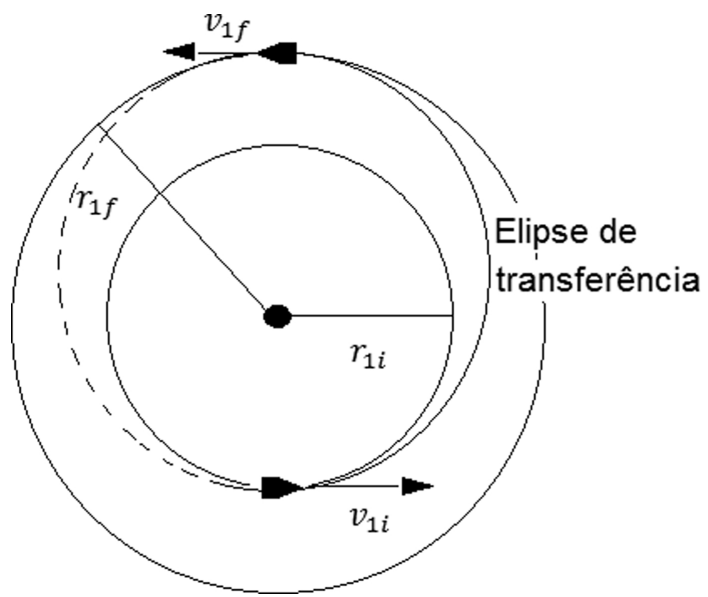

Figura 3: Manobra Hohmann que leva de uma órbita circular de raio $r_{1 i}$ para uma de raio $r_{1 f}$. 
circular. Assim teremos $\Delta v^{\prime}=v_{1 f}-v_{e}^{\prime}$ onde

$$
\begin{aligned}
& -\frac{k}{r_{1 i}+r_{1 f}}=m v_{e^{\prime}}^{2} / 2-\frac{k}{r_{1 f}} \Rightarrow v_{e}^{\prime} \\
& =\sqrt{\frac{2 k}{m} \frac{r_{1 i}}{r_{1 f}\left(r_{1 i}+r_{1 f}\right)}}
\end{aligned}
$$

A manobra Hohmann utiliza dois impulsos e é a mais efetiva energeticamente. Se o tempo for um fator importante, podemos também utilizar uma manobra bielíptica, que utiliza três impulsos e duas elipses de transferência 11, vide Figura 4. Para um exemplo moderno, o acoplamento entre a Estação Espacial Internacional (ISS) e a nave Soyuz (que leva suprimentos e transporta os astronautas nos trajetos de ida e volta a ISS) dura cerca de seis horas quando utilizada um conjunto de manobras Hohmann e transferência bielíptica. Se utilizada apenas manobras Hohmann, o acoplamento pode levar até quarenta e oito horas, contadas a partir do lançamento da Soyuz [12]. A manobra bielíptica faz uso do efeito Oberth 11] que diz que para um impulso $\Delta \vec{v}$ o ganho em energia cinética é maior no perigeu. De fato, como:

$$
\Delta T=\frac{(\vec{v}+\Delta \vec{v})^{2}}{2}-\frac{\vec{v}^{2}}{2}=\frac{\Delta \vec{v}^{2}}{2}+\vec{v} \cdot \Delta \vec{v}
$$

teremos que, para um certo $\Delta \vec{v}$, o ganho de energia cinética é maior quanto maior $\vec{v}$, o que ocorre no perigeu (e, incidentalmente, quanto mais baixa a órbita). Considere que vamos utilizar uma transferência bielíptica para sairmos de uma órbita circular $r_{1}$ para uma de raio $r_{2}>r_{1}$. Utilizamos nessa manobra três impulsos ao invés dos dois utilizados na Hohmann: inicialmente passamos de uma órbita

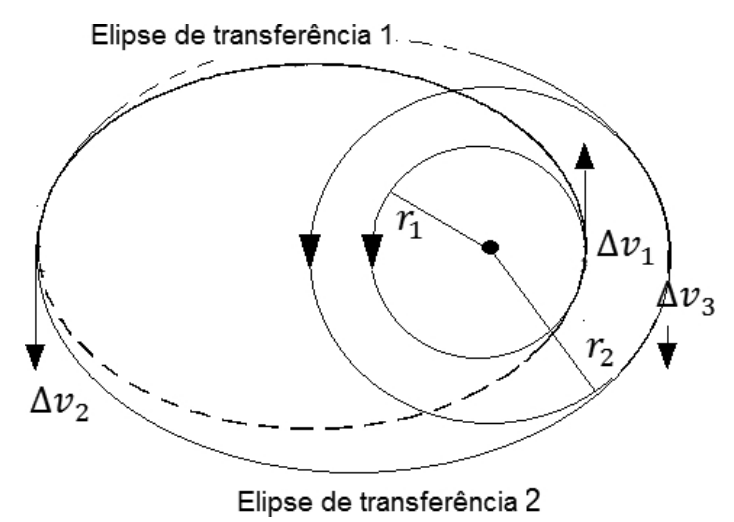

Figura 4: Impulsos necessários na manobra bielíptica. Estão presentes duas elipses de transferência, no lugar da única necessária para a manobra Hohmann. circular $r_{1}$ para uma elíptica de perigeu $r_{1}$ e um certo apogeu $r_{a}>>r_{2}$. Para isso damos o impulso:

$$
\Delta v_{1}=v_{1 p}-v_{i}=\sqrt{2 G M_{T} \frac{r_{a}}{r_{1}\left(r_{1}+r_{a}\right)}}-\sqrt{\frac{G M_{T}}{r_{1}}}
$$

onde $v_{1 p}$ é a velocidade que o corpo deve possuir no perigeu $r_{1}$ de uma órbita elíptica e $v_{i}$ é a velocidade do corpo na órbita circular de raio $r_{1}$. Ao atingirmos o apogeu $r_{a}$ dessa órbita um novo impulso nos leva à outra órbita elíptica com perigeu $r_{2}$ :

$$
\begin{aligned}
\Delta v_{2} & =v_{2 a}-v_{1 a}=\sqrt{2 G M_{T} \frac{r_{2}}{r_{a}\left(r_{2}+r_{a}\right)}} \\
& -\sqrt{2 G M_{T} \frac{r_{1}}{r_{a}\left(r_{1}+r_{a}\right)}}
\end{aligned}
$$

onde $v_{2 a}$ é a velocidade que o corpo deve ter na segunda elipse de transferência e $v_{1 a}$ é a velocidade no apogeu na primeira elipse de trasferência. Por último, ao atingirmos o perigeu $r_{2}$ um último impulso transforma essa órbita elíptica numa circular de raio $r_{2}$. O impulso sendo igual a:

$$
\Delta v_{3}=v_{f}-v_{2 p}=\sqrt{\frac{G M_{T}}{r_{2}}}-\sqrt{2 G M_{T} \frac{r_{a}}{r_{2}\left(r_{2}+r_{a}\right)}}
$$

onde $v_{f}$ é a velocidade de um corpo em órbita circular de raio $r_{2}$ e $v_{2 p}$ a velocidade no perigeu da segunda elipse de transferência. Esse último impulso é claramente um desperdício de energia e é a razão da manobra ser menos eficiente que a de Hohmann. Das equações para $\Delta v_{2}$ vemos que uma opção é aumentar cada vez mais $r_{a}$ (que é a razão de escolhermos $r_{a}>>r_{2}$ ). Entretanto, isso aumenta o tempo gasto na manobra e eventualmente ela pode não ser favorável se o tempo gasto for similar a Hohmann.

Por fim, se for necessário uma mudança no plano orbital (mudança na inclinação $I$ ) devemos mudar a direção do vetor velocidade e fornecer um impulso $\Delta \vec{v}=\vec{v}_{1 f}-\vec{v}_{1 i}$. Note que $v_{1 f}=v_{1 i}$ se a mudança for apenas na inclinação (caso contrário alteraremos a altitude da nave), onde as velocidades são tangentes às órbitas, que fazem entre si um ângulo $\alpha$, cujo valor nos dá a magnitude do impulso: $\Delta v=\sqrt{v_{1 i}^{2}+v_{1 f}^{2}-2 v_{1 f}^{2} v_{1 i}^{2} \cos \alpha}$. Mudanças na inclinação requerem menor energia se realizadas no apogeu, onde $v_{1 i}$ é mínimo. 


\section{Conclusão}

Os soviéticos ficaram definitivamente para trás na corrida espacial após a morte de Korolev em 1966. Contribuiu para esse fato outra morte, em 1967, do cosmonauta Vladimir Komarov a bordo da Soyuz 1. Ainda assim os russos conseguiram realizar com sucesso o primeiro rendezvous entre duas espaçonaves tripuladas com a Soyuz 4 e 5 em 1969. Um feito que não teve a merecida atenção pois, um ano antes, os americanos conseguiram um feito ainda mais ambicioso ao enviarem a Apollo 8 para um voo de circunavegação Lunar. Uma série de problemas com o novo foguete $N 1$ minou as esperanças russas de enviarem uma missão tripulada à Lua antes dos americanos, o que não os impediu de serem bem sucedidos com artefatos não tripulados. De fato, um deles estava na Lua quando a Apollo 11 realizou a primeira alunissagem tripulada, em 1969 [2]. Pouco tempo depois, o interesse público pela corrida espacial se desvaneceu, exceto talvez por um breve momento com a quase tragédia da missão Apollo 13 [3.

A disputa pela primazia do espaço se encerrou com outro célebre rendezvous, realizado em 1975 entre uma espaçonave americana, a Apollo 18, e a nave russa Soyuz 19. Após o acoplamento e abertura das escotilhas, o cosmonauta Alexei Leonov, o primeiro homem a caminhar no espaço, cumprimentou Thomas Stafford e Deke Slayton, um dos pioneiros astronautas do programa Mercury, que ainda não havia ido ao espaço devido à problemas cardíacos. Tal ato, realizado a centenas de kilômetros acima da superfície terrestre selou, ao menos nesse palco, o fim da guerra fria.

\section{Referências}

[1] D. Scott, A. Leonov and C. Toomey, Two Sides of the Moon (St. Martin Press, New York, 2004).

[2] D.K. Slayton and M. Kassut, Deke! (Tom Doherty, New York, 1994)

[3] G. Kranz, Failure Is Not An Option (Simon \& Schuster, New York, 2000).

[4] B. Aldrin and K. Abrahan, Magnificent Desolation (Randon House, New York, 2009).

[5] E. Cernan and D. Davis, Last Man on the Moon (St. Martin Press, New York, 1999).

[6] A. Worden and F. French, Falling to Earth (Smithsonian Books, Washington, 2011).
[7] E.E. Audrin, Line of Sight Guidance Techniques for Manned Orbital Rendez-Vous. Phd Thesis, M.I.T, 1963.

[8] S.T. Thornton and J.B. Marion, Classical Dynamics of Particles and Systems (Brooks/Cole - Thomson Learning, Belmont, 2004).

[9] http://quest.nasa.gov/space/teachers/ liftoff/math.html

[10] R.A. Braeunig, http://www.braeunig.us/space/ index.htm

[11] http://mmae.iit.edu/ mpeet/Classes/ MMAE441/Spacecraft/441Lecture21.pdf

[12] http://www.esa.int/ 